\title{
A review of models used for understanding epileptic seizures
}

\author{
$\underline{\text { J. M. Dunn }}^{a}$ and R.S. Anderssen ${ }^{b}$ \\ ${ }^{a}$ School of Mathematical and Geospatial Sciences, RMIT University, GPO Box 2476, Melbourne, \\ Victoria, 3001 \\ ${ }^{\mathrm{b}}$ CSIRO Mathematics, Informatics and Statistics, \\ Canberra, Australia \\ Email: jessica.dunn@rmit.edu.au
}

\begin{abstract}
The development and application of mathematics, fostered by a mathematics-in-industry study group (MISG) activity, is a key outcome of the challenges posed when aiming to answer the questions raised by real-world problems. Mathematics-in-industry study groups, which now thrive internationally, bring together mathematicians from tertiary institutions and industries to provide a stimulating environment for the discussion, analysis and solutions of such problems.
\end{abstract}

Mathematics-in-industry study groups (MISGs) have been actively providing a forum for the development and application of mathematics for industrial modelling projects. When considering industrial mathematical applications, it is important to recognise that industrial processes work because of their inherent robustness (de Hoog, 2009). Consequently, the formulation and analysis of the simple models which encapsulate the associated robustness are an essential part of industrial mathematical modelling. This approach to modelling allows for the uncertainty in modelling to be managed by a relatively simple model that describes the process in question and can capture an answer the industrial partners can exploit operationally. At the MISG 2010 at RMIT University, in a study of the recovery of the location and time of the initiation of an epileptic episode, the importance of the joint utilisation of electroencephalogram (EEG) and functional MRI (fMRI) data became apparent for:

1. Reducing the uncertainty about the location and time of the commencement of an epileptic seizure.

2. Providing an understanding about the neuronal mechanism causing epilepsy as a means to reduce the uncertainty around minimizing associated risks.

In particular, the process of sychronisation forms a foundation for the understanding of epileptic episodes. Synchronisation occurs when two or more process couple to harmonise in a similar manner. It is a naturally occurring physical, physicochemical and biological phenomenon. Examples include superconductivity, neuronal behaviour in the brain, adjacent clocks, circadian rhythm in animals and plants and the fundamental processes in population dynamics. In this paper, the modelling of synchronisation with the simple and exactly solvable Kuramoto model is discussed. Generalisations of this model are reviewed which more correctly reflects the known behaviour of synchronisation in the brain. A strategy for the joint inversion of the EEG and fMRI data is proposed.

Keywords: Epilepsy, industrial mathematics, Kuramoto model, synchronisation 


\section{The Phenomenon of Sychronisation}

Synchronisation occurs when two or more process couple to operate in a similar manner. This phenomenon is closely related to the concepts of "constructive interference" and "resonance" and is a naturally occurring physical, physicochemical and biological phenomenon. Biologically, it is the mechanism that controls the beating of the heart with the synchronization of the signalling coming from the heart's pacemaker (Winfree, 1980; Okuda and Kuramoto, 1991) and the process responsible for brain activity.

Brain activity is an extraordinarily complex dynamic signalling and switching process. It is also a highly organized, robust and error-correcting activity where processes are normally robustly and stably synchronised. Insel (2010) hypothesized that fundamental shifts in brain activity, such as those occurring in mental illnesses, is a type of failure of the stability of multiple synchronisation paths in the brain. This hypothesis can be extended to include the abnormal brain activity arising in epilepsy where malfunctions in areas of normal synchronisation are observable (Velazquez, 2005). Figure 1(a) illustrates electroencephalogram (EEG) recordings which are representative of the brain activity occurring prior to, at and during a seizure (Kim et al., 2009).

Currently, the observation of brain activity over time is mostly done through EEG recordings (Figure 1(a)) and when viewed together with fMRI data, which shows the localisation of blood flow changes in the brain (Figure 1(b)), uncertainty about the location and the time of commencement of an epileptic seizure is reduced. Electroencephalogram (EEG) recordings accurately identify the time at which synchronous epileptic activity commences within the brain and highlights the rapid onset oscillatory phase occurring during an epileptic event. As is clear from such plots, as shown in Figure 1(a), the rapid onset of the highly oscillatory phases can be viewed as occurring through some major synchronous activity within the brain.

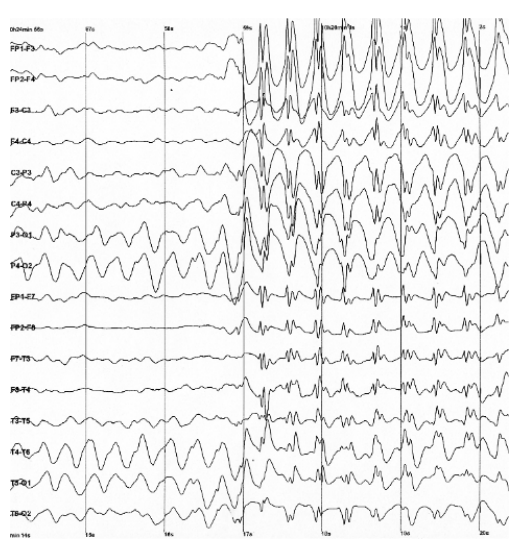

(a) EEG Data showing onset of an epileptic event.

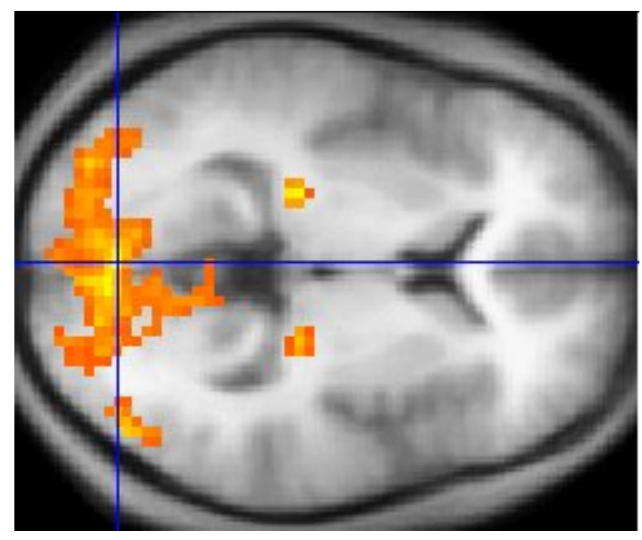

(b) fMRI Data showing localization of blood flow and metabolic changes.

Figure 1. Brain activity measurements: time (EEG) and location (fMRI) data.

Synchronisation phenomenon behave universally similar in almost all natural occurring oscillations and can be described by the dynamical system known as the Kuramoto model (Kuramoto, 1975). However, in modelling such synchronization, the appropriateness of the Kuramoto model (Kuramoto, 1984) has not been explicitly examined. This model has been indirectly linked with epilepsy, mentioned as an example of a complex network which synchronises. The Kuramoto model models the behaviour of $\mathrm{N}$ coupled oscillators. The formulation of this model was motivated by the earlier research of Winfree (1967) who, among other things, proved that the degree of synchronisation depended on the degree of coupling between the oscillators. 


\section{The Kuramoto Model}

As highlighted by a number of authors (Strogatz, 2001; Cumin and Unsworth, 2007; Acebron et al., 2005), it was Winfree (1967) who, in order to explain the observed behaviour of populations of biological oscillators, formulated a model consisting of a system of nearly identical and coupled limit-cycle oscillators. Winfree (1967) went on to prove whether a system behaves incoherently or synchronises depending on the degree of coupling; namely, incoherent when the coupling was small corresponding to a large spread in frequencies of the oscillators; partial synchronisation when the coupling exceeded a threshold; and fully locked phase and amplitude synchronisation for strong coupling. An illustration of this can be found in Figure 2 in Strogatz (2001).

A lucid discussion of the Kuramoto model as a simple paradigm for the study of synchronisation can be found in Acebron et al. (2005). The importance of having simple models is reflected in the following comment by Velazquez (2005) "... the classic Kuramoto model $\cdots$ a model of simplicity that captures very importance aspects $\cdots$ a lesson for those who think that simple, naive models have little practical use in real life" and discussed from an industrial mathematics perspective by de Hoog (de Hoog, 2009).

It was Kuramoto (1984) who formalized mathematically the observations of Winfree (1967) in a form that yielded a mathematical framework in which to successfully study synchronization. In addition, he proposed a simple and exactly solvable model of collective synchronization which highlighted explicitly the intuitive observations for Winfree (1967) and thereby validated the appropriateness of his general mathematical model. An insightful discussion of the mathematics of the Kuramoto model can be found in Strogatz (2000). Because of its utility and appropriateness in modelling, for example, lasers, heart beat and brain activity, it is often cited as an example of a methodology for exploring complex networks (Strogatz, 2001).

For $N$ oscillators, the general form of the model proposed by Kuramoto (1984), with $t$ denoting time, is given by

$$
\dot{\theta}_{j}(t)=\omega_{j}+\sum_{i=1}^{N} \Gamma_{j i}\left(\theta_{i}-\theta_{j}\right), \quad \theta_{j}=\theta_{j}(t), \quad \dot{\theta}_{j}=\frac{d \theta_{j}}{d t}, \quad j=1, \cdots, N,
$$

where $\theta_{j}$ and $\omega_{j}$ denote, respectively, the phase and frequency of oscillator $j$ with the function $\Gamma_{i j}\left(\omega_{j}-\right.$ $\omega_{i}$ ) defining the nature of the coupling occurring between oscillators $i$ and $j$.

The interesting special case of equally weighted, all-in-all, purely sinusoidal coupling, for which $\mathrm{Ku}-$ ramoto Kuramoto (1984) gave an analytic solution, is given by

$$
\dot{\theta}_{j}(t)=\omega_{j}+\frac{K}{N} \sum_{i=1}^{N} \sin \left(\theta_{i}-\theta_{j}\right), \quad j=1, \cdots, N .
$$

As explained in Strogatz (2000), for highlighting the nature of the synchronization implicit in this model, Kuramoto introduced the concept of an order parameter to characterize the degree of the synchronization ("the collective rhythm" of the population of oscillators) occurring. The complex order parameter takes the form

$$
r(t) \exp (i \psi(t))=\frac{1}{N} \sum_{j=1}^{N} \exp \left(i \theta_{j}\right)
$$

with $r(t)$ and $\psi(t)$ tracking the phase coherence and average phase of the population.

Using this definition for the order parameter, Kuromoto was able to rewrite, after some algebra, it in the following more compact (mean-field) form

$$
\dot{\theta}_{j}(t)=\omega_{j}+K r(t) \sin \left(\psi-\theta_{j}\right), \quad j=1, \cdots, N .
$$

Even though the oscillators are interacting, this equation shows that the coupling is through the order parameter's phase coherence $r$ and the average phase $\psi$. It follows from (4) that, as the time $t$ progresses with synchronization occurring, the phases $\theta_{j}$ are pulled towards $\psi$ rather than each other and the strength of the coupling is given by $K r$. 


\section{SYNCHRONISATION AND BRAIN FUNCTION}

The Kuramoto model, as discussed above, is now playing an increasing role in the modelling of neuronal activity (Kuramoto, 1991; Han et al., 1995; Frank et al., 2000). However, the model's appropriateness as a model for the study of epilepsy remains an open question. The current emphasis on the study of epilepsy is focussed on techniques for the recovery of information jointly from EEG and fMRI data, with independent component analysis (ICA) receiving considerable attention (Calhoun et al., 2005; Eichele et al., 2009).

Various modelling aspects have been pursued but with only an indirect connection to the Kuramoto model. Kim et al. (Kim and Robinson, 2007, 2008; Kim et al., 2009) used delay differential equations in their study of the evolution, spreading and suppression dynamics of epilepsy. In Kim et al. (2009) a passing comment is made about "Kuramato type all-in-all coupling" without specifically citing a publication by Kuramoto. However, they do cite papers related to Parkinson's disease where the Kuramoto model is specifically mentioned. Interestingly, in brain activity studies, the Kuramoto model is playing a quite important role in the study of desynchronization in the context of pulse-based desynchronization techniques for the treatment of Parkinson's disease (Dolan et al., 2005; Majtanik et al., 2006; Moazami-Goudarzi et al., 2008). However, it should be mentioned that such investigations give no insight about the nature of the initiation of the synchronization in either epilepsy or Parkinson's disease.

Kuramoto (1991) has examined collective synchronization of pulse-coupled oscillators and excitable units, where the concept of "all-in-all mutual connection" is introduced. This has relevance for neuronal modelling, because the neuronal interaction and communication is a pulsative interaction process.

\subsection{Generalisations of the Kuramoto Model}

As explained by Cumin and Unsworth (Cumin and Unsworth, 2007) and others, from a brain activity perspective, the weakness of the simple Kuramoto model of equation (1) is that it assumes that the coupling between each pair of oscillators contributing to the synchronization are all the same. Clearly, as Cumin and Unsworth proposed, for the modelling of the synchronization in brain activity, an appropriate generalisation of the Kuramoto model takes the form

$$
\dot{\theta}_{j}(t)=\omega_{j}+\frac{1}{N} \sum_{i=1}^{N} K_{i j}(t) \sin \left(\theta_{i}-\theta_{j}\right), \quad j=1, \cdots, N,
$$

where the different levels of coupling between oscillators are not the same and change with the progression of the time $t$. The clear advantage of this model is that the synchronization can be modelled over the whole brain by setting the $K_{i j}(t)$ to be zero for the times when the coupling between the $i$-th and $j$-th oscillators is zero. Interestingly, through the introduction of an explicit dependence on the time $t$ into the coupling function $\Gamma_{i j}\left(\theta_{i}-\theta_{j}\right)$ of Equation (1), the Cumin and Unsworth generalization is also a generalization of the original mean-field model of Kuramoto (1984). In Cumin and Unsworth (2007), extensive computational analysis of a coupled four-component model was performed in order to assess the utility of the generalization.

A number of authors have examined the utility of the Cumin-Unsworth generalization for the modelling of neuronal activity. The utility of the generalization is acknowledged in Velazquez et al. (2007), who suggest its application to earlier work on desynchronization (Majtanik et al., 2006). It is explicitly exploited in Lin and Lin (2009); Ghosh et al. (2009), where the generalization is used to study neuronal synchronization in the brain. A connection to the circadian rhythm is made in Granada et al. (2009).

A key question of how to utilise the Kuramoto model in the study of epilepsy has been considered by a number of authors. Velazquez et al. (2007) examined the recovery of information using phase response curves (PRCs).

\subsection{Joint Inversion of the EEG and fMRI data}

The underlying inverse problem of recovering information about an epileptic seizure involves both a qualitative and quantitative aspect: 
(i) Is the nature of a seizure such that it is correct to assume that it starts at a specific location and time with the brain with the ramification of that being the EEG recording of a seizure (i.e. a recording of brain activity before at and after the commencement of the seizure)?

(ii) Assuming that the answer to (i) is yes, how can the EEG and fMRI data be jointly utilised to identify the location and time?

The strategy suggested here to perform a location and time identification consists of the following two steps:

(a) Within an EEG recording of a seizure, identify the features $f_{\mathrm{EEG}}(i), i=1,2, \ldots, I$, which highlight the changing structure in the time series data at the time a seizure occurs.

(b) With respect to the identified features $f_{\mathrm{EEG}}(i), i=1,2, \ldots, I$, search for similar features in the fMRI data, and use the location and time of that fMRI feature as the location and time for the epileptic event identified in the EEG.

Clearly the success of this process will depend on choosing a form for the features which will facilitate the search in (b). In addition, by identifying a number of features $f_{\mathrm{EEG}}(i), i=1,2, \ldots, I$, the probability is reasonably high that at least one of them will see a similar structure in the fMRI data. Note that, the features should be as simple as possible as it is more likely to be robust.

\section{REFERENCES}

Acebron, J., L. Bonilla, C. Vicente, F. Ritort, and R. Spigler (2005, JAN). The Kuramoto model: A simple paradigm for synchronization phenomena. Rev. Mod. Phys. 77(1), 137-185.

Calhoun, V., T. Adali, M. Stevens, K. Kiehl, and J. Pekar (2005, APR 1). Semi-blind ICA of fMRI: a method for utilizing hypothesis-derived time courses in a spatial ICA analysis. Neuroimage 25(2), $527-538$.

Cumin, D. and C. P. Unsworth (2007, FEB 15). Generalising the Kuramoto model for the study of neuronal synchronisation in the brain. Physica D-Nonlinear Phenonmena 226(2), 181-196.

de Hoog, F. R. (2009). Why are simple models often appropriate in industrial mathematics? 18th World IMACS/MODSIM Congress, Cairns, July 13-17 Proceedings, 23-36.

Dolan, K., M. Majtanik, and P. Tass (2005, NOV 1). Phase resetting and transient desynchronization in networks of globally coupled phase oscillators with inertia. Physica D-Nonlinear Phenonmena 211(12), 128-138.

Eichele, T., V. D. Calhoun, and S. Debener (2009, JUL). Mining EEG-fMRI using independent component analysis. Int. J. Psychophysiology 73(1, Sp. Iss. SI), 53-61.

Frank, T., A. Daffertshofer, C. Peper, P. Beek, and H. Haken (2000, SEP 15). Towards a comprehensive theory of brain activity: Coupled oscillator systems under external forces. Physica D-Nonlinear Phenonmena 144(1-2), 62-86.

Ghosh, A., D. Roy, and V. K. Jirsa (2009, OCT). Simple model for bursting dynamics of neurons. Phys. Rev. E 80(4, Part 1).

Granada, A., R. M. Hennig, B. Ronacher, A. Kramer, and H. Herzel (2009). Phase Response Curves: Elucidating the Dynamics of Coupled Oscillators. Volume 454 of Methods in Enzymology, pp. 1-27.

Han, S., C. Kurrer, and Y. Kyuramoto (1995, OCT 23). Dephasing and Bursting in Coupled Neural Oscillators. Phys. Rev, Lett. 75(17), 3190-3193.

Insel, T. R. (2010, April). Faulty circuits. Scientific American 302, 44-51. 
Kim, J. W., J. A. Roberts, and P. A. Robinson (2009, APR 21). Dynamics of epileptic seizures: Evolution, spreading, and suppression. J. Theo. Bio. 257(4), 527-532.

Kim, J. W. and P. A. Robinson (2007, MAR). Compact dynamical model of brain activity. Phys. Rev. E 75(3, Part 1).

Kim, J. W. and P. A. Robinson (2008, MAY). Controlling limit-cycle behaviors of brain activity. Phys. Rev. $E$ 77(5, Part 1).

Kuramoto, Y. (1975). Int. symp. on mathematical problems in theoretical physics. In H. Araki (Ed.), Lect. N. Phys., Volume 39, pp. 420422. Springer, New York.

Kuramoto, Y. (1984). Chemical Oscillations, Waves and Turbulence. Berlin: Springer Verlag.

Kuramoto, Y. (1991, MAY). Collective Synchronization of Pulse-Coupled Oscillators and Excitable Units. Physica D 50(1), 15-30.

Lin, C. and M.-m. Lin (2009, AUG). The mathematical research for the Kuramoto model of the describing neuronal synchrony in the brain. Communications in Nonlinear Science and Numerical Simulation 14(8), 3258-3260.

Majtanik, M., K. Dolan, and P. A. Tass (2006, OCT). Desynchronization in networks of globally coupled neurons with dendritic dynamics. J. Bio. Phys. 32(3-4), 307-333.

Moazami-Goudarzi, M., J. Sarnthein, L. Michels, R. Moukhtieva, and D. Jeanmonod (2008, JUL). Enhanced frontal low and high frequency power and synchronization in the resting EEG of parkinsonian patients. Neuroimage 41(3), 985-997.

Okuda, K. and Y. Kuramoto (1991, DEC). Mutal entrainment between populations of coupled oscillators. Prog. Theo. Phys. 86(6), 1159-1176.

Strogatz, S. (2000, SEP 1). From Kuramoto to Crawford: exploring the onset of synchronization in populations of coupled oscillators. Physica D 143(1-4), 1-20.

Strogatz, S. (2001, MAR 8). Exploring complex networks. Nature 410(6825), 268-276.

Velazquez, J. (2005, DEC 15). Brain, behaviour and mathematics: Are we using the right approaches? Physica D-Nonlinear Phenomena 212(3-4), 161-182.

Velazquez, J. L. P., R. F. Galan, L. G. Dominguez, Y. Leshchenko, S. Lo, J. Belkas, and R. G. Erra (2007, DEC). Phase response curves in the characterization of epileptiform activity. Phys. Rev. E 76(6, Part $1)$.

Winfree, A. (1967). Biological rhythms and behavior of populations of couple oscillators. J. Theo. Bio. 16(1), 15-\&.

Winfree, A. (1980). The Geometry of Biological Time. New York: Springer Verlag. 